\title{
Our Experience with Glomus Tumor
}

\section{Anantheswar YN*, Reddy S, Ashok BC, Srikanth V, Joyce J, Kumar P, Ritu , Harish and Dinkar \\ Manipal Hopsitals, India}

*Corresponding author: Dr Anantheswar YN, Department of Plastic Surgery, Manipal Hospital, No.98, Rustombagh, Old Airport Road, Bengaluru-560017, India, Tel: +919845016595; Email: ananth1961gmail.com

\section{Research Article}

Volume 2 Issue 1

Received Date: April 19, 2018

Published Date: May 16, 2018

DOI: $10.23880 /$ ijtps- 16000115

\section{Abstract}

We present a study of sixteen patients with glomus tumor- including clinical presentation, diagnosis, treatment and follow up. This rare but interesting tumor is not easily visible, palpable or imaged on radiography. Though the patient is symptomatic early, diagnosis is usually delayed. The treating clinician needs to have a high index of suspicion. Complete cure involves excision of the tumor.

Keywords: Glomus tumor; Difficulty in diagnosis; MRI; Transungual excision under microscope and prevention of deformity

\section{Introduction}

Glomus tumors are rare hamartomas consisting of endothelium lined vascular spaces (Sucquet Hoyer canal) surrounded by glomus cells [1-4]. These are benign neoplasms arising from the glomus body located in the dermis and related to thermoregulation. Though commonly found in the subungual region, they have been reported in several other organs [5]. They may be either solitary or multiple and are commonly seen in females.

The classic triad of symptoms includes localized tenderness, paroxysmal pain and cold sensitivity [6]. Despite this knowledge, it becomes difficult to diagnose this condition due to the nonspecific and varied clinical presentation. Imaging modality of Magnetic Resonance aids in the diagnosis.

\section{Materials and Methods}

This retrospective study includes sixteen patients who presented over a period of five years from April 2011 to May 2016 at our institution and underwent surgery for subungual glomus tumour. Their hospital records were analyzed in detail with respect to clinical features, imaging studies, operative records, histopathology reports and follow up.

Fifteen patients had a glomus tumour in a finger (index, middle finger or thumb) and one in great toe. The patients were in the age group of 29 to 59 years of which ten were female and six were male. All of them had pain in the digit as the presenting complaint. Four patients could appreciate a swelling in the affected digit, two patients had the characteristic bluish discoloration spot below the nail. Based upon clinical impression of glomus tumour, diagnosis was established using MRI of the digit.

All patients underwent surgical excision of the tumor, six patients under regional block and rest under general anaesthesia. The procedure was carried out under an operating microscope after applying a tourniquet to the limb. The excised lesion was sent for histopathological examination.

The surgical treatment involved a transungual approach with a dorsal incision on the tip of the digit. The nail was elevated in a hinged manner and the bluish 


\section{International Journal of Transplantation \& Plastic Surgery}

tinged swelling was identified, in the nail bed. The tumour was dissected free and a wide excision of $0.25 \mathrm{~mm}$ all around margin was carried out. The nail was perforated at few places to allow the drainage of subungual fluid collection and repositioned back into the nail bed. The wound was closed with 6-0 prolene and soft occlusive dressing with plaster of paris slab was given.

\section{Results}

After the surgery, all patients were comfortable and discharged on the same day. Histopathological examination confirmed diagnosis of glomus tumor in all. Post- operatively, all patients were symptom free, without any complications each follow up visit on $3^{\text {rd }}$ day, 2 weeks, 3 months, 6 months and one year later. Out of sixteen patients one patient presented with recurrence at the same site, three years after the surgery. During the previous surgery MRI did not show the lesion. However excision was done on high index of clinical suspicion. When he underwent surgery for recurrence, MRI was documented clear oval lesion.

\begin{tabular}{|c|c|c|c|c|c|c|c|c|c|}
\hline Name & Age & Sex & Location & Clinical features & On examination & MRI & HPE & Follow up & Final outcome \\
\hline NT & 29 & M & IF & P,S & T, W & Y & C & NC & Nc \\
\hline RB & 47 & F & Thumb & P, Ti & T, B & Y & C & NC & Nc \\
\hline SL & 35 & F & Thumb & P, S & T & Y & C & NC & Nc \\
\hline PL & 29 & F & 1st toe & P & T & Y & C & NC & Nc \\
\hline AI & 32 & F & Thumb & P & T & Y & C & NC & Nc \\
\hline AR & 45 & F & IF & P & T & Y & C & NC & Nc \\
\hline JP & 51 & M & MF & P & T & Y & C & NC & Nc \\
\hline MO & 34 & F & MF & P & T & Y & C & NC & Nc \\
\hline AJ & 28 & F & IF & P & T & Y & C & NC & Nc \\
\hline CK & 37 & F & IF & P,S & T & Y & C & NC & Nc \\
\hline JL & 39 & F & MF & P & T & Y & C & NC & Nc \\
\hline KV & 21 & F & IF & P & T & Y & C & NC & Nc \\
\hline NV & 42 & M & MF & P,S & T & Y & C & NC & Nc \\
\hline RS & 29 & M & Thumb & P, Ti & T & Y & C & NC & Nc \\
\hline RP & 47 & M & MF & P & T & Y & C & NC & Nc \\
\hline RR & 58 & M & RF & P, Ti & T,B & Y & C & Recurrence & Nc \\
\hline
\end{tabular}

Table 1: Patient characteristics.

Abbreviations: IF: Index finger; MF: Middle finger; P: Pain; S: Swelling; Ti: Tingling: T: Tenderness; W: Whitish nail; B: Bluish nail bed; Y: Yes; C: Confirmed; NC: No complaints; Nc: No complications.

\section{Discussion}

Glomus tumor is a rare and benign tumor, arising from the subcutaneous glomus apparatus. It was first discovered in 1812 by Wood and described by Masson in his monologue Les glomus cutanes de l 'Homme in 1935 [7]. The etiology (though considered unknown), could be due to trauma or a hamartoma. It commonly affects patients between the third and fifth decade, with a female preponderance, as was also seen in our study.

The patients usually present with non specific symptoms to various specialties and are diagnosed late. The common symptoms include pain, sensitivity to touch, cold sensitivity, bluish discoloration (Figure 1) and nail deformity. Pain is the dominating symptom and one which is most discomforting to the patient. The tumor is sensitive to temperature changes, resulting in contraction of myofilaments and rise in intracapsular pressure, which is transmitted via the unmyelinated nerve fibers leading to the perception of pain [8].

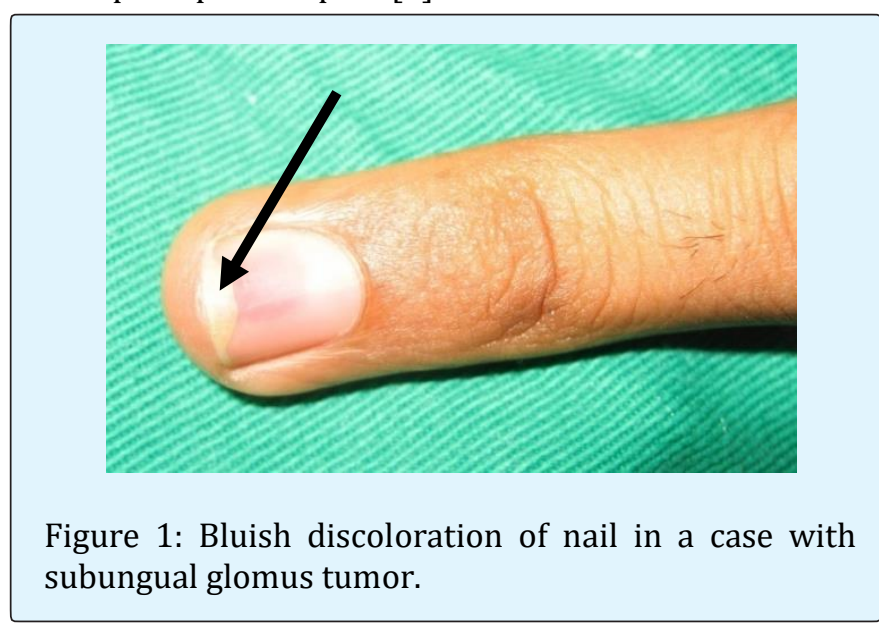




\section{International Journal of Transplantation \& Plastic Surgery}

Diagnosis is made largely on clinical basis. Two signs, named after Love and Hildreth, are helpful [9]. The Love's sign is positive when there is severe localized tenderness on applying pressure with a pinhead and relieved on removing the pressure. The Hildreth's sign is performed by elevating the patient's arm and then tying a tourniquet to the arm which is inflated to $250 \mathrm{mmHg}$. This result in reduction or abolition of pain in the digit with glomus tumor and the pain reappears when the tourniquet is deflated.

Imaging helps in confirming the diagnosis. X-ray is the least specific and shows underlying cortical bone thinning or erosion [10]. This however is seen in long standing or large lesions. On ultrasonography these tumors appear as well circumscribed hypoechoic masses. However as the presence of nail generates artifacts, its utility is limited in small or flat lesions. Doppler Ultrasound picks up these hypo echoic masses better. MRI, a non invasive technique, is most useful in evaluating these tumors. It can detect lesions up to $2 \mathrm{~mm}$ [11]. They appear hypo dense on T1, hyper dense on $\mathrm{T} 2$ and are enhanced on gadolinium contrast. It is also useful to detect residual and recurrent lesions. Al-Qattan, et al. reported a sensitivity of $90 \%$ and positive predictive value of $97 \%$ in their series of 42 patients [12]. In our study, MRI was a helpful modality to detect and confirm these tumors (Figure 2).

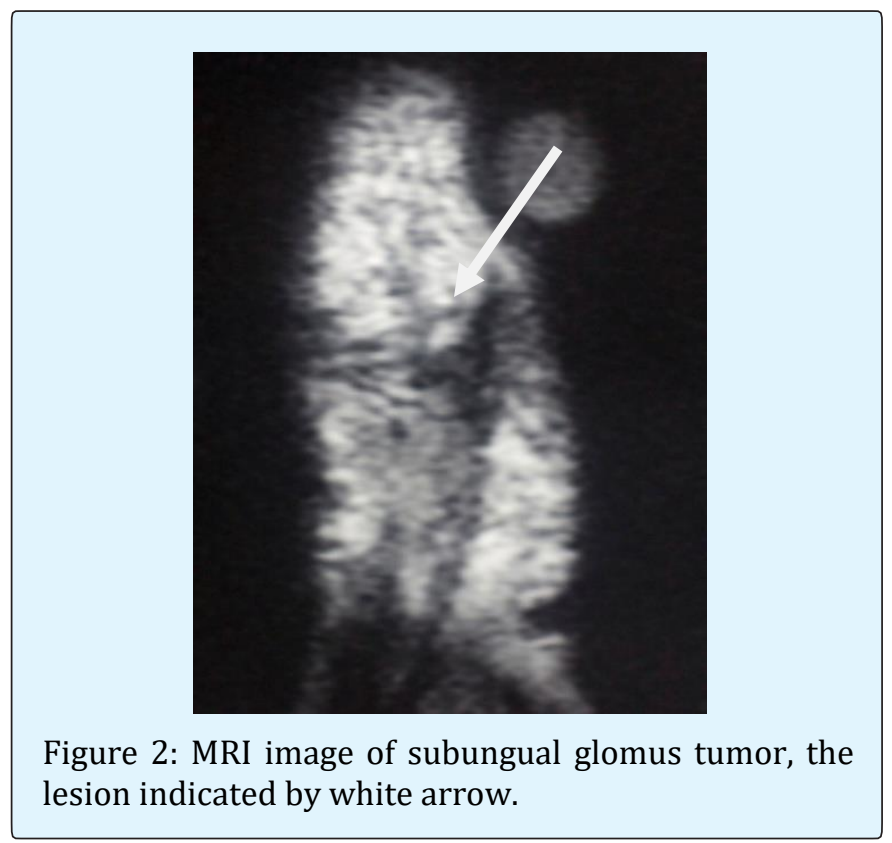

Histology shows specialized smooth muscle cells (glomus cells), dilated vascular spaces, nerve fibers and mast cells. They can be categorized into three types (solid, glomangiomas, glomangiomyomas) according to the predominant component. Solid glomus tumors (75\%) predominating in glomus cells, Glomangiomas (20\%), with more of vascular component and Glomangiomyomas (5\%) with predominance of vascular structures and smooth muscle cells [13]. Histochemical staining is positive for vimentin, alpha smooth muscle actin and negative for desmin [14].

Treatment is primarily surgical. There are two approaches, transungual and lateral Keyser -Littler approach [15] (Figure 3). The transungual approach has certain advantages, which include a better view enabling complete excision. If the tumor involves the nail matrix, an eponychial flap has to be raised for a clearer view.

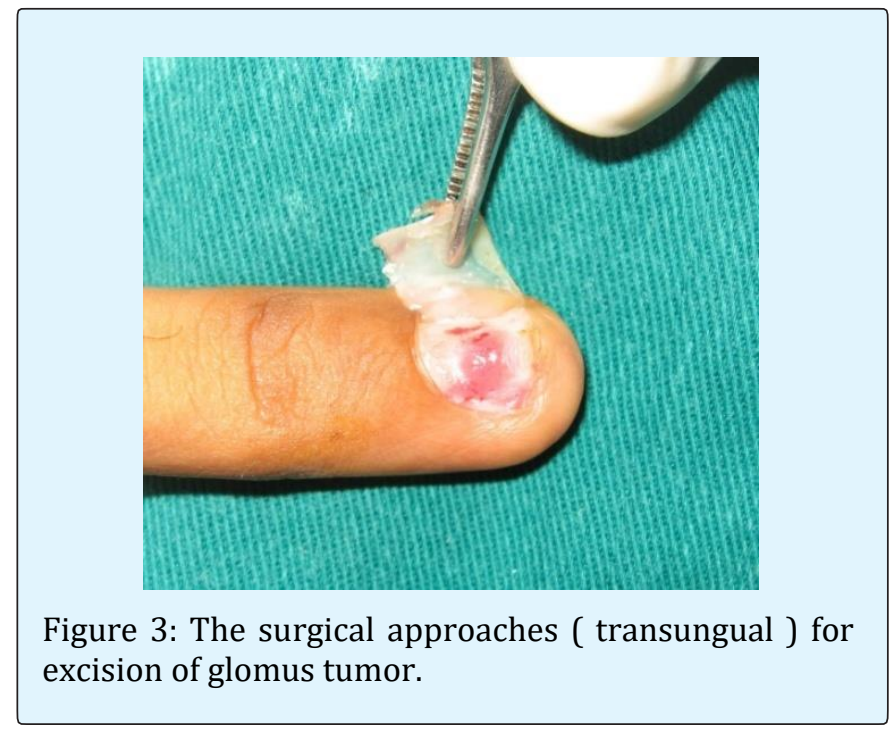

\section{An algorithmic approach to GT:}

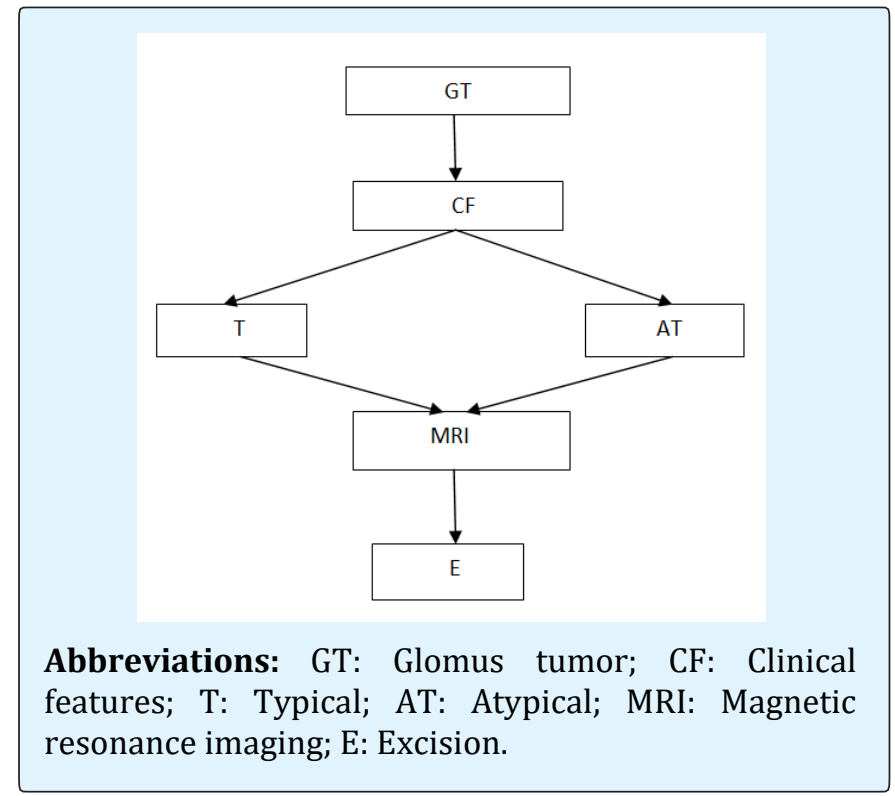




\section{International Journal of Transplantation \& Plastic Surgery}

The complications of surgery are nail deformity and recurrence. Recurrence has been recently attributed to the presence of satellite lesions [8], which are not recognized during surgery and regrow with time and cause pain. The nail deformity could be due to surgical insult of the nail bed or adhesions between matrix and proximal nail fold. Raising of nail in a hinged fashion and making a few perforations before reposition will prevent subungual hematoma and adhesions. Repositioning of the nail plate prevents adhesions between the eponychium and matrix, keeps the wound covered, favours nail bed repair and reduces pain during change of dressings [16]. Li, et al. have made use of autologous fat grafting to repair defect in nail bed [17]. We used prolene 6.0 to close the defect and found satisfactory result. One patient presented with recurrence after 3 years and underwent excision without any complications (Figure 4).

Based on our study we have formulated an algorithmic approach to a patient presenting with glomus tumour.

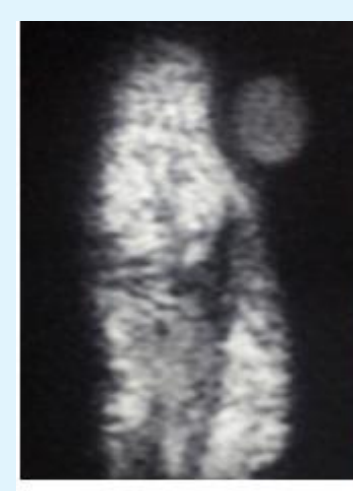

a

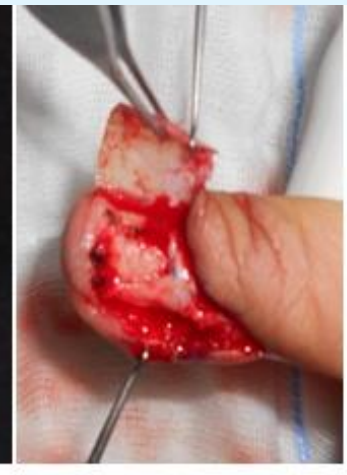

b

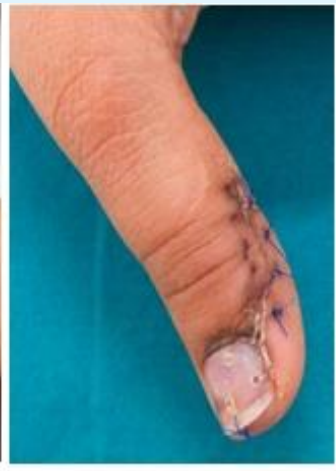

C

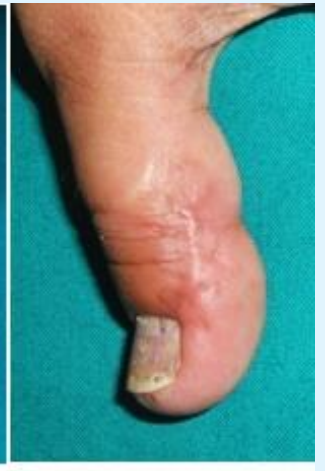

d

Figure 4: Patient with subungual glomus tumor in thumb with figure a. showing MRI imaging of lesion, b. post excision of the tumor, c. closure of defect and d. post operative well healed scar.

\section{Conclusion}

Glomus tumor owes its importance to two facts- firstly the characteristic and disturbing pain as the clinical presentation, secondly, the difficulty in diagnosis. Owing to the first characteristic, it becomes important to remove this benign tumor; however, the second characteristic makes the task difficult. In all our cases we were able to diagnose patients based on experience, awareness and a high index of suspicion. We confirmed the diagnosis with MRI. During surgery we adopted a transungual approach which facilitated thorough examination of the region, enabled complete excision of the tumor and prevention of nail deformity. The post operative follow up was unremarkable in all our patients without any residual tumor, except one patient presented with recurrence after 3 years and underwent excision without any complications.

\section{References}

1. Carroll RE and Berman AT (1972) Glomus tumors of the hand: Review of the literature and report of
Twenty eight cases. J Bone Joint Surg Am 54(4): 691703.

2. Schiefer TK, Parker WL, Anakwenze OA, Amadio PC, Inwards CY, et al. (2006) Extradigital glomus tumors: A 20 year experience. Mayo Clinic Proceedings 81(10): 1337-1344.

3. Rettig AC, Strictland JW (1977) Glomus tumor of the digits. J Hand Surg Am 2(4): 261-265.

4. Mathis WH, Schulz (1948) Roentgen diagnosis of glomus tumors. Radiology 51(1).

5. Gaertner EM, Steinberg DM, Huber M, Hayashi T, Tsuda N, et al. (2000) Pulmonary and Mediastinal Glomus Tumors:Report of five cases including a Pulmonary Glomangiosarcoma: A clinicopathologic Study with Literature review. Am J Surg Pathol 24(8): 1105-1114.

6. Gombos Z, Zhang PJ (2008) Glomus Tumor. Arch Pathol Lab Med 132(9): 1448-1452. 


\section{International Journal of Transplantation \& Plastic Surgery}

7. Murray MR, Stout AP (1942) The glomus tumorInvestigation of its distribution and the identity of its "epithelioid" cell. Am J Pathol 18(2): 183-203.

8. Grover C, Khurana A, Jain R, Rathi V (2013) Transungual surgical excision of glomus tumor. J Cutan Aesthet Surg 6(4): 196-203.

9. Hazani R, Houle JM, Kasdan ML, Wilhelmi BJ (2008) Glomus tumors of the hand. Eplasty 8: e48.

10. Glazebrook KN, Laundre BJ,Schiefer TK, Inwards CY (2011) Imaging features of glomus tumors. Skeletal radiology 40(7): 855-862.

11. Drape JL, Idy-Peretti I, Goettmann S, Guerin-Surville H, Bittoun J (1996) Standard and high resolution imaging of glomus tumors of toes and fingertips. J Am Acad Dermatol 35(4): 550-555.

12. Al- Qattan MM, Al-Namla A, Al-Thunayan A, Al- Subhi F, El-Shayeb AF (2005) Magnetic Resonance Imaging in the Diagnosis of Glomus Tumors of the Hand. J Hand Surg Eur 30(5): 535-540.
13. Tsuneyoshi M, Enjoji M (1982) Glomus tumor. A Clinicopathologic and electron microscopic study. Cancer 50(8): 1601-1607.

14. Nobuyuki H, Noriaki K, Takahiro T, Kazuaki T, Hiroko $\mathrm{N}$, et al. (1997) Malignant Glomus Tumor: A Case Report and review of the Literature. Am J Surg Pathol 21(9):1096-1103.

15. Vasisht B, Watson HK, Joseph E, Lionelli GT (2004) Digital Glomus Tumors: A 29 Year Experience with a Lateral Subperiosteal Approach. Plast Reconstr Surg 114(6): 1486-1489.

16. Tada H, Hirayma T, Takemitsu Y (1994) Prevention of postoperative nail deformity after subungual glomus resection. J Hand Surg Am 19(3): 500-503.

17. Li TS, Choong MY, Wu CI, Chen HC, Chung KC (2011) Autologous fat graft to restore nail bed contour following resection of a subungual glomus. J Hand Surg Am 36(4): 726-728. 